\title{
Determination of the accuracy of imaging methods to evaluate bone in maxilla for implant placement- An invitro study
}

\author{
Venkat Reddy Marakala ${ }^{1}$, Shylaja Allu², Vinil Chaitanya Pittala ${ }^{3}$ \\ 1,2 Assistant Professor, ${ }^{3}$ Senior Resident, ${ }^{1,3}$ Dept. of Dentistry, ESIC Medical College \& Hospital, Hyderabad, \\ Telangana, ${ }^{2}$ Dept. of Prosthodontics, Mallareddy Dental College for Women, Hyderabad, Telangana, India
}

*Corresponding Author:

Email: markala77@yahoo.com

\begin{abstract}
Introduction: Dental implants are now treatment of choice in replacing of missing teeth. Treatment outcomes with dental implants are predictable and favourable. Long term implant survival is dependent on proper implant planning and case selection. Precision in dental implant placement is the sine qua non for dental implant success. Radiographic examinations are the only non-invasive technique for visualizing the bone topography, volume and location of vital structures like mandibular canal and maxillary sinuses. In this study we have tried to evaluate commonly used radiographic modalities in implant planning, orthopantomograph (OPG) and computed tomography (CT) and tried to compare their accuracy with direct measurements with skull sectioning at predetermined maxillary first molar region.

Materials and Methods: Ten dry human skulls were subjected to OPG, CT and skull sectioning respectively. Measurements on height and width of the alveolar bone were noted at the maxillary first molar region and results were compared.

Results: The mean height of alveolar bone measured using OPG, CT and Skull sectioning was found to be $12 \mathrm{~mm}, 9.4 \mathrm{~mm}$ and $9.3 \mathrm{~mm}$ respectively. The mean value of width of alveolar bone measured using CT and skull sectioning was found to be equal at $10.3 \mathrm{~mm}$. Additionally, the mean rate of magnification for OPG was found to be $40 \%$, whereas for CT it was just $2 \%$.
\end{abstract}

Keywords: Orthopantomography, Computed tomography, Dental Implants, Maxillary sinus.

\section{Introduction}

Dental implants replace missing teeth permanently without compromising the structure of adjacent teeth but require higher precision in placing them. Before placing a dental implant, dentists must visualize 3-dimensionally, among others the two most important structures, the location of maxillary sinuses in maxilla and the mandibular nerve canal in mandible. Dental implant placement in posterior maxilla is particularly challenging due to the presence of maxillary paranasal sinuses and poor quality of bone in that region. ${ }^{1}$ Understanding the location of maxillary sinuses is of vital importance for placement of maxillary implants as the sinus perforation may cause subsequent antral infections result in implant failure. ${ }^{2}$

Another problem frequently encountered in implant placement is the lack of bone quantity. Normally, a thin plate of cortical bone separates tooth apices and the maxillary floor, but in some posterior teeth only a thin mucosal layer is present between them. There is a natural tendency towards increasing the volume of maxillary sinus during life due to osteoclastic activity in the schneiderian membrane. This extension of maxillary sinus combined with rapid alveolar bone resorption due to loss of tooth in the posterior maxilla can result in causing pneumatization of the sinus by resorbing bone within a few months. ${ }^{3}$ In such cases, maxillary sinus floor elevation surgery may be considered which will require a closer look to its anatomical variations. ${ }^{4}$

Radiography is the only technique which can assess the extent of pneumatization of maxillary sinus and thus plays a vital role in implant dentistry. Imaging helps in optimal placement of the implants and enhances its success, both short and long term and of all subsequent stages of the procedure. ${ }^{5}$

Computed tomography (CT)/Cone beam computed tomography (CBCT) for dental implant surgery planning is being carried out routinely by the dentists. It helps in exact preoperative assessment of the available bone volume and to determine the appropriate length, position and angulation of the planned implants. ${ }^{6}$ There is no doubt that the Computed tomographic examinations are effective means of evaluating the bone volume present below the maxillary sinuses. ${ }^{7}$

But higher cost of Computed tomographic scans may be a major deterrent factor for its use in implant imaging. Also, Compared to other radiographic examinations, CT scans deliver a relatively high dose of radiation to the patient. ${ }^{8}$ While this is not usually a problem for a single scan, patients who need to undergo repeated tests can be subjected to a significant level of radiation. Of late, the cone beam $\mathrm{CT}$ has alleviated risk of radiation to a certain degree. ${ }^{9}$

Orthopantamograph (OPG) is another imaging modality which is both inexpensive and also widely available. OPG causes much lower dose of radiation exposure than CT/ CBCT to the patients, thus making it a much safer option. It is instrumental in making preliminary estimates of maxillary sinus. But, OPG can have significant unequal image distortion which is the main reason for its limitation as definitive pre-surgical planning tool. ${ }^{10}$

In this study we wish to determine the magnification and compare the efficacy of CT and OPG in 
measurement of height of the alveolar bone from the floor of the maxillary sinus to the crest to the alveolar ridge at the first molar region of dried skulls with direct measurement by skull sectioning. We will also compare the efficacy of CT in measuring the width of the available crestal alveolar bone at the first molar region of dried skulls.

\section{Materials and Methods}

Ten dry human skulls have been used in this study. The skulls have been evaluated at the maxillary first molar region bilaterally, ending up with a total of twenty evaluated samples. An impression, for each skull that was used in the present study, was taken with alginate impression material and subsequently a working cast was made. This cast was used for the fabrication of radiographic stent. The undercut areas in the working cast were arbitrarily analyzed and were blocked off using modeling wax.

Fabrication of radiographic stent: Casts of maxillary arches of the skulls was made with dental stone. A Radiographic stent was made using clear acrylic material on each of the working cast. A hole was drilled in the first molar region bilaterally, and a metal ball measuring the size of $4.76 \mathrm{~mm}$ diameter was stuck using adhesive in these holes. These metallic balls were used as position indicators and also as magnification assessment guide. These radiographic stents were initially used for panoramic radiography and were retained till further radiographic examination with CT. The same stents were later used for CT after replacing the metallic balls with Gutta Percha.

OPG procedure: Planmeca Proline EC Panoramic $\mathrm{X}$-ray machine was used. The stent was then transferred to the skull and placed in position. Each skull along with the stent in place was mounted on a tripod stand and stabilized using modeling wax. The skull was positioned upright with Frankfort horizontal line parallel to floor and the sagittal line of the skull perpendicular to floor. The maxillary arches were placed within the focal trough of the machine as per the position indicating devices of the manufacturer. The skull along the radiographic stent was then subjected to radiation with exposure parameters of $70 \mathrm{kvp}, 9 \mathrm{~mA}$ for $18 \mathrm{sec}$.

The measurements were made bilaterally on each skull from crest of the alveolar ridge to the floor of the maxillary sinus using the Romexis viewer programme.

Procedure for CT: The Computed Tomographic scanner used for radiographic evaluation of maxilla was 64-slice scanner (Siemens - Somatom Sensation 64, Germany). The CTD volume applied for the procedure was $14.10 \mathrm{mgy}$. The scan time was 11 seconds, MAS - 90, $\mathrm{KV}-120$; field of view was $180 \mathrm{~mm}$ with Slice thickness of $0.75 \mathrm{~mm}$ and reconstruction with $50 \%$ overlap.

All the skulls were subjected to CT after OPG evaluation. The radiographic stents that were initially used in OPG were again placed on the skulls after replacing the metallic balls with Gutta Percha. After the initial topography (AP) of the entire skull, axial sections were obtained in the region of maxilla.

Each Skull was positioned on the table in supine position with the skull resting within the head rest. The skull was stabilized using straps to avoid motion artifacts in the scan. Topographic view of CT scan was taken with zero degree Gantry angle. Axial sections of the mandible were taken from the topographic view of CT scan parallel to the maxillary arches at $0.75 \mathrm{~mm}$ thickness of slice with no overlap. These axial sections were imported to the Dentascan dental software. The slices of axial sections were subjected to reformat into panoramic and paraxial sections as reference image. The position of the maxillary first molar region was located by the grey image of gutta-percha within the radiographic stent from the paraxial and the panoramic view.

The sections of the acquired data with $0.75 \mathrm{~mm}$ reconstruction, with $50 \%$ overlap, was located into the dental CT software provided by the vender Siemens, Germany. Four sets of data were obtained. The first image being sagittal MIP (maximum intensity production) as planned for the maxilla, performed with horizontal line parallel to the alveolar margin. The paraxial sagittal images were obtained from right maxillary tuberosity to the left maxillary tuberosity. The paraxial sections are contiguous sections depicting the buccal and palatal aspects of the maxilla. Measurements were performed using these images after identifying the floor of the maxillary sinus at the point vertically above the crest of the maxillary alveolar ridge in the region of first maxillary molar as identified from the gutta-percha of the radiographic stent.

Procedure for sectioning of skull: All the skulls that were subjected to $\mathrm{CT}$ and panoramic radiographic evaluation were sectioned. The radiographic stents that were initially used in panoramic radiography and then again used in CT were also used here to the site for sectioning. The Gutta-percha in the stents was removed by spoon excavator and the site for sectioning the maxilla of the skull in the first molar region was marked with a marker pen. Consequently the maxilla of each skull was sectioned bilaterally in the first molar region as marked earlier using a fretsaw. The measurements were made bilaterally on each skull from crest of the alveolar ridge to the floor of the maxillary sinus using digital vernier calipers. Measurements thus obtained from direct measurements were compared with those of the OPG and CT.

\section{Results}

This study included ten human skulls which were subjected to OPG, CT and direct measurement by sectioning of maxilla at the maxillary first molar region. The parameters that were measured with these techniques are as follows:

1. Height of the available bone in maxilla from floor of the maxillary sinus to the crest of the alveolar ridge, 
with both OPG and CT and were compared with direct measurements made by skull sectioning.

2. The width of the available crestal bone at first molar region was measured by $\mathrm{CT}$ and compared with the direct measurements made by skull sectioning.

While taking OPG, a radiographic stent embedded with a metallic ball of known diameter $(4.76 \mathrm{~mm})$, was fabricated for each skull. In an attempt to compensate for the magnification in OPG, the Actual height of the alveolar bone was calculated by multiplying the measured height of the alveolar bone in OPG with magnification factor. The magnification factor was calculated individually, by dividing the Known
Diameter of Metal Ball $(4.76 \mathrm{~mm})$ with the diameter of the metal ball measured on that OPG.

Actual height of Alveolar bone = Magnification factor $\mathrm{X}$ Height of alveolar bone measured in OPG

Magnification factor $=\frac{\text { Known Diameter of Metal Ball }(4.76 \mathrm{~mm})}{\text { Diameter of the metal ball measured on OPG in } \mathrm{mm}}$

Comparison of measurements in vertical bone height from crest of the alveolar ridge to the floor of the maxillary sinus was done with the OPG, CT and skull sectioning. The details are given in the Table 1.

Table 1: Bone Height Measured with OPG, CT and Skull-Sectioning

\begin{tabular}{|c|c|c|c|c|c|c|}
\hline \multirow{2}{*}{ Name } & \multicolumn{2}{|c|}{$\begin{array}{c}\text { Bone height } \\
\text { measured with } \\
\text { OPG in mm }\end{array}$} & \multicolumn{2}{c|}{$\begin{array}{c}\text { Bone height } \\
\text { measured with CT } \\
\text { in mm }\end{array}$} & \multicolumn{2}{c|}{$\begin{array}{c}\text { Bone height measured } \\
\text { with Skull-Sectioning in } \\
\text { mm }\end{array}$} \\
\cline { 2 - 7 } & Right & Left & Right & Left & Right & Left \\
\hline Skull A & 5.2 & 4.7 & 4.7 & 3.4 & 4.3 & 3.2 \\
\hline Skull B & 8.6 & 12.3 & 6.7 & 15.5 & 6.5 & 16 \\
\hline Skull C & 7.6 & 6.4 & 4.3 & 5.6 & 4.1 & 5.5 \\
\hline Skull D & 7.1 & 7.6 & 4.9 & 3.9 & 5 & 4 \\
\hline Skull E & 14.3 & 15.2 & 14.5 & 13 & 15 & 12 \\
\hline Skull F & 13 & 15 & 10 & 10 & 10 & 10 \\
\hline Skull G & 19 & 20 & 17 & 16 & 17 & 16 \\
\hline Skull H & 17 & 15 & 13 & 10 & 13 & 10 \\
\hline Skull I & 10 & 9 & 7 & 5 & 7 & 5 \\
\hline Skull J & 16 & 17 & 13 & 11 & 12 & 10 \\
\hline
\end{tabular}

The minimum height of the alveolar bone as measured in OPG in the sample is $4.7 \mathrm{~mm}$ and the maximum height is $20 \mathrm{~mm}$. The mean value of the height of the alveolar bone as measured in OPG is found to be $12 \mathrm{~mm}$ with a standard deviation of $4.7 \mathrm{~mm}$. The details are given in the Table 1, Table 2, and Graph 1.

Table 2: Statistical Analysis of Bone Height Measured with OPG, CT and Skull-Sectioning using ANOVA with Post Hoc Bonferroni Correction

\begin{tabular}{|l|c|c|c|c|c|}
\hline \multicolumn{1}{|c|}{ Methods } & Mean & SD & N & p-value & post-hoc test \\
\hline $\begin{array}{l}\text { Height in Skull- } \\
\text { sectioning }\end{array}$ & 9.3 & 0.46 & 20 & $<0.001$ & $\begin{array}{c}\text { OPG }> \\
\text { Skull-sectioning } \\
\text { and CT }\end{array}$ \\
\cline { 1 - 4 } Height in CT & 9.4 & 0.45 & 20 & & \\
\hline Height in OPG & 12 & 0.47 & 20 & & \\
\hline
\end{tabular}

Graph 1: Mean height with skull sectioning, CT \& OPG

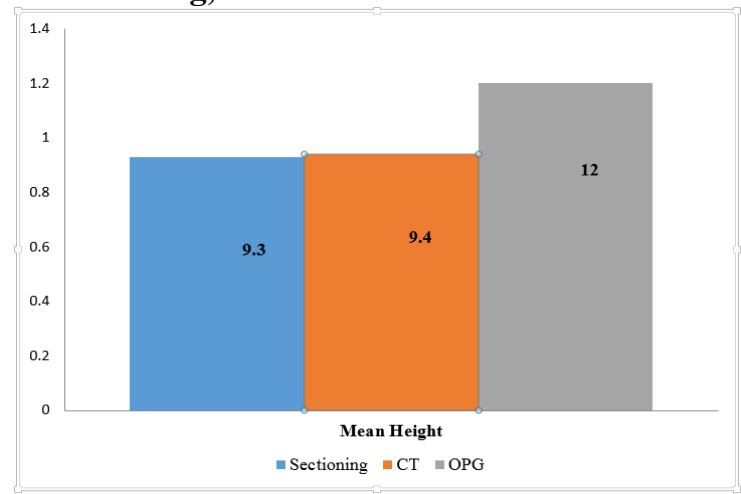


The minimum height of the alveolar bone as measured in CT scans in the sample is $3.4 \mathrm{~mm}$ and the maximum height is $17 \mathrm{~mm}$. The mean value of the height of the alveolar bone as measured in CT is found to be $9.4 \mathrm{~mm}$ with a standard deviation of $4.5 \mathrm{~mm}$. The details are given in the Table 1, Table 2 and Graph 1.

The minimum height of the alveolar bone as measured by sectioning the maxilla in the sample is 3.2 $\mathrm{mm}$ and the maximum height is $17 \mathrm{~mm}$. The mean value of the height of the alveolar bone as measured by sectioning the maxilla is found to be $9.3 \mathrm{~mm}$ with a standard deviation of $4.6 \mathrm{~mm}$. The details are given in the Table 1, Table 2 and Graph 1.

The Repeated measures analysis of variance (ANOVA) with post hoc bonferroni correction was used to test the group difference and also pair wise variable differences. The $\mathrm{p}$ value for the ANOVA is less than 0.001 indicating the statistically significant difference in the mean alveolar height within three study groups. $(\mathrm{p}<$ 0.001). The details are given in the Table 2.

Thus, Post hoc analysis was done to evaluate significant inter-group comparisons. There was

significant higher mean alveolar height measured with OPG than that of the mean alveolar height measured with CT and sectioning the maxilla. No significant difference is seen between the mean alveolar height measured with CT and that of the direct measurement by sectioning the maxilla. The details are given in the Table 2 .

On comparison using paired t-test, the height of the alveolar bone as measured in CT and that measured by sectioning the maxilla, the mean value of the height of the alveolar bone as measured in CT was found to be $9.4 \mathrm{~mm}$ with a standard deviation of $4.5 \mathrm{~mm}$. and the mean value of the height of the alveolar bone as measured by sectioning the maxilla was found to be $9.3 \mathrm{~mm}$ with a standard deviation of $4.6 \mathrm{~mm}$. The mean difference between the two study groups is $0.1 \mathrm{~mm}$ with standard deviation of $0.05 \mathrm{~mm}$ and $\mathrm{p}$ value being 0.4 , which is not statistically significant difference $(\mathrm{p}=0.4)$. The details are given in the Table 3, Table 4 and Graph 2.

Table 3: Paired t-test for Comparison among Bone Height Measured with OPG, CT and Skull-Sectioning

\begin{tabular}{|c|c|c|c|c|}
\hline Methods & Mean & $\mathbf{N}$ & SD & p-value \\
\hline Height in Skull- sectioning & 9.3 & 20 & 0.46 & \multirow[t]{2}{*}{0.4} \\
\hline Height in CT & 9.4 & 20 & 0.45 & \\
\hline Height in Skull- sectioning & 9.3 & 20 & 0.46 & \multirow[t]{2}{*}{$<0.001$} \\
\hline Height in OPG & 12 & 20 & 0.47 & \\
\hline Height in CT & 9.4 & 20 & 0.45 & \multirow[t]{2}{*}{$<0.001$} \\
\hline Height in OPG & 12 & 20 & 0.47 & \\
\hline
\end{tabular}

Table 4: Mean differences between groups using Paired t-tests

\begin{tabular}{|c|c|c|c|c|c|c|}
\hline \multirow[t]{3}{*}{ Parameters } & \multicolumn{2}{|c|}{$\begin{array}{c}\text { Paired } \\
\text { Differences }\end{array}$} & \multirow{2}{*}{\multicolumn{2}{|c|}{$\begin{array}{l}\text { 95\% Confidence } \\
\text { Interval of the } \\
\text { Difference }\end{array}$}} & \multirow[t]{3}{*}{$\mathrm{t}$} & \multirow[t]{3}{*}{$\mathrm{df}$} \\
\hline & Mean & SD & & & & \\
\hline & & & Lower & Upper & & \\
\hline Sectioning Height \& CT Height & -0.01 & 0.05 & -0.0326 & 0.013602 & -0.86069 & 19 \\
\hline Sectioning Height \& OPG Height & -0.27 & 0.23 & -0.37235 & -0.16065 & -5.26973 & 19 \\
\hline CT Height \& OPG Height & -0.26 & 0.21 & -0.35552 & -0.15848 & -5.45964 & 19 \\
\hline
\end{tabular}

Graph 2: Mean height with Skull- Sectioning and CT

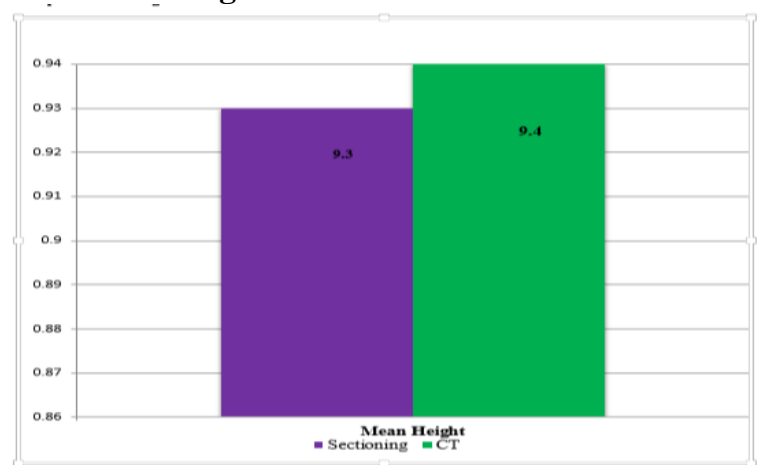




\section{Graph 3: Mean height with Skull-sectioning and OPG}

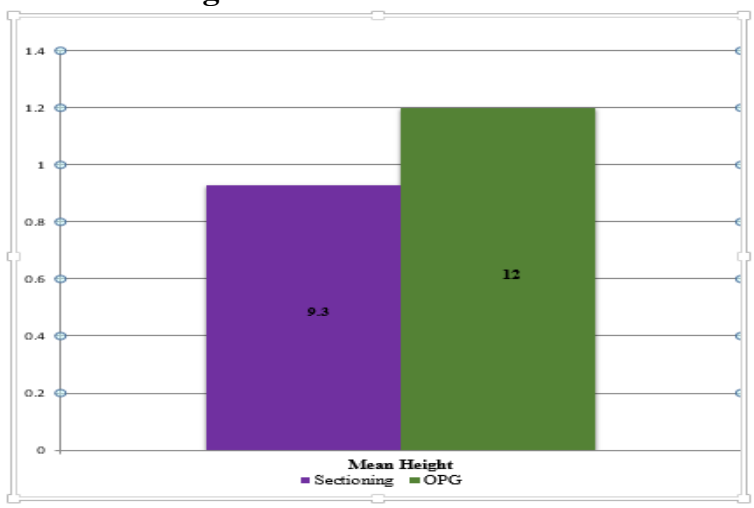

\section{Graph 4: Mean height with CT \& OPG}

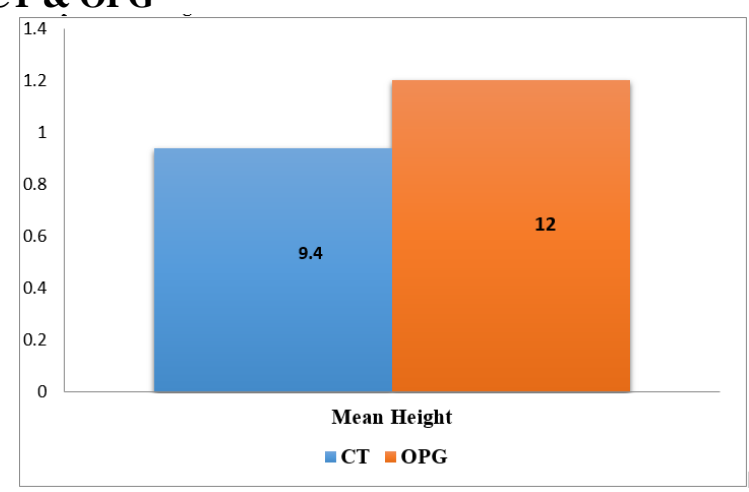

On comparison using paired t-test, the height of the alveolar bone measured with OPG and that measured by sectioning the maxilla, the mean value obtained with OPG is $12 \mathrm{~mm}$ with a standard deviation of $4.7 \mathrm{~mm}$ and the mean value of the height of the alveolar bone as measured by sectioning the maxilla was found to be $9.3 \mathrm{~mm}$ with a standard deviation of $4.6 \mathrm{~mm}$. The mean difference between these two study groups is $2.7 \mathrm{~mm}$ and standard deviation 0.23 and with $\mathrm{p}$ value less than 0.001 , which is a statistically significant difference. The details are given in the Table 3, Table 4 and Graph 3.

On comparison using paired t-test, the height of the alveolar bone measured with, OPG and CT, the mean value obtained with OPG is $12 \mathrm{~mm}$ with a standard deviation of $4.7 \mathrm{~mm}$ and the mean value of the height of the alveolar bone as measured in CT is found to be 9.4 $\mathrm{mm}$ with a standard deviation of $4.5 \mathrm{~mm}$. The mean difference between the two study groups is $2.6 \mathrm{~mm}$ and standard deviation 0.21 and with $p$ value less than 0.001 , which is statistically significant difference. The details are given in the Table 3, Table 4 and Graph 4.

Additionally the mean width of the available crestal bone was measured in CT and compared with direct measurement made on the skull.

The width of the available crestal bone as measured in CT ranged from $4.9 \mathrm{~mm}$ to $18.7 \mathrm{~mm}$. The minimum width of the available crestal bone as measured in CT in the sample is $4.9 \mathrm{~mm}$ and the maximum width is 18.7 $\mathrm{mm}$. The mean value of CT width was found to be 10.3 $\mathrm{mm}$ with a standard deviation of $3.2 \mathrm{~mm}$. The details are given in the Table 5, Table 6 and Graph 5.

Table 5: Bone Width measured with CT and Skull-Sectioning

\begin{tabular}{|c|c|c|c|c|}
\hline Name & \multicolumn{2}{|c|}{$\begin{array}{c}\text { Bone Width } \\
\text { measured with CT in } \\
\text { mm }\end{array}$} & $\begin{array}{c}\text { Bone Width measured } \\
\text { with Skull-Sectioning in } \\
\text { mm }\end{array}$ \\
\cline { 2 - 5 } & Right & Left & Right & Left \\
\hline Skull A & 5.5 & 4.9 & 5 & 4.5 \\
\hline Skull B & 10.1 & 10 & 10.3 & 10 \\
\hline Skull C & 7.2 & 8.1 & 7.1 & 8 \\
\hline Skull D & 18.7 & 12 & 19 & 12 \\
\hline Skull E & 12 & 12 & 11 & 12 \\
\hline
\end{tabular}




\begin{tabular}{|c|c|c|c|c|}
\hline Skull F & 8 & 9 & 8 & 9 \\
\hline Skull G & 12 & 10 & 12 & 10 \\
\hline Skull H & 11 & 9 & 10 & 10 \\
\hline Skull I & 10 & 8 & 10 & 9 \\
\hline Skull J & 15 & 14 & 15 & 15 \\
\hline
\end{tabular}

Table 6: Statistical Analysis of Bone Width Measured with CT and Skull-Sectioning using Paired t test

\begin{tabular}{|l|c|c|c|c|}
\hline & Mean & N & SD & p-value \\
\hline $\begin{array}{l}\text { Bone Width in Skull- } \\
\text { sectioning }\end{array}$ & 1.03 & 20 & 0.34 & .87 \\
\cline { 1 - 4 } Bone Width in CT & 1.03 & 20 & 0.32 & \\
\hline
\end{tabular}

\section{Graph 5: Mean width}

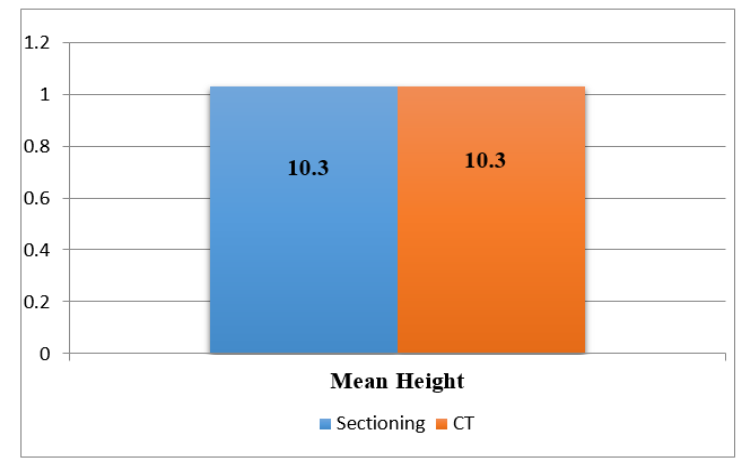

The width of the available crestal bone as measured directly on the skull ranged from $4.5 \mathrm{~mm}$ to $19 \mathrm{~mm}$. The minimum width of the available crestal bone as measured directly on the skull is $4.5 \mathrm{~mm}$ and the maximum width is $19 \mathrm{~mm}$. The mean value of the available crestal bone as measured directly on skull was found to be $10.3 \mathrm{~mm}$ with a standard deviation of 3.4 $\mathrm{mm}$. The details are given in the Table 5, Table 6 and Graph 5.
Paired t test was used to test the group difference and also pair wise variable differences, if any. The $p$ value for the Paired $\mathrm{t}$ test was 0.87 indicating the statistically insignificant difference in the mean crestal width within the two study groups. There was no significant difference in the mean crestal width as measured in $\mathrm{CT}$ and that of the width of the crestal bone as measured directly on the skull using sectioning $(\mathrm{p}=0.87)$. The details are given in the Table 6 .

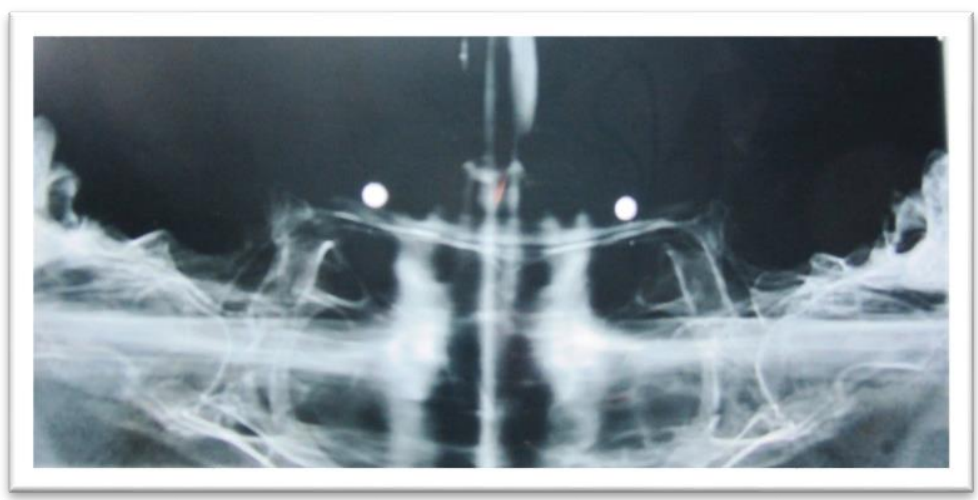

Fig. 1: OPG of a skull showing metal balls at the maxillary $1^{\text {st }}$ molar region 


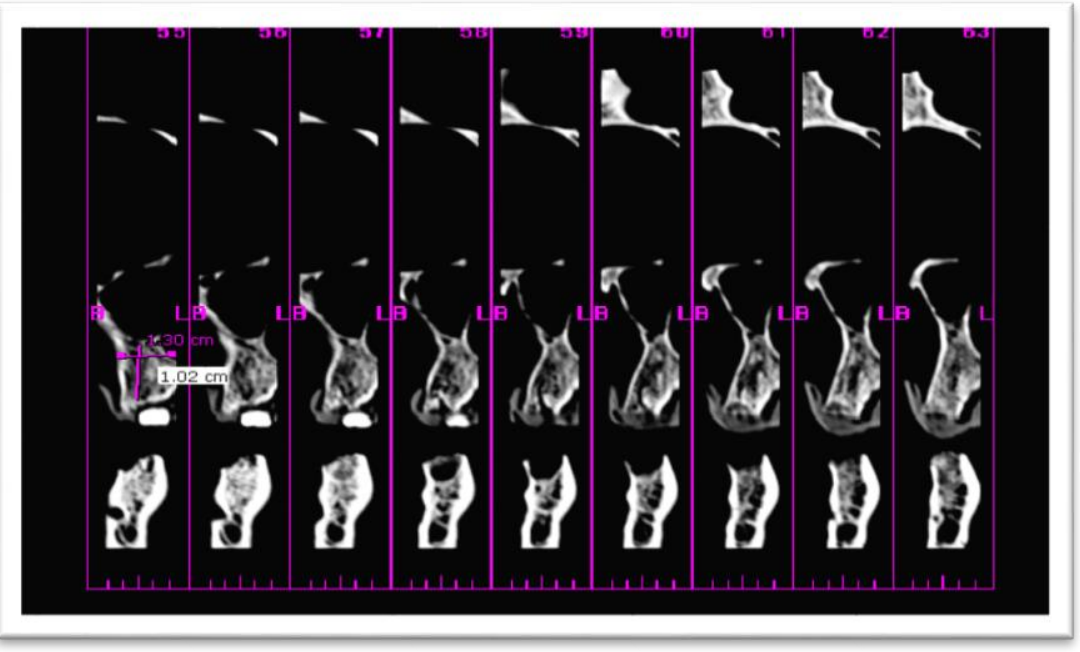

Fig. 2: Paraxial CT of a skull showing gutta-percha at the maxillary $1^{\text {st }}$ molar region

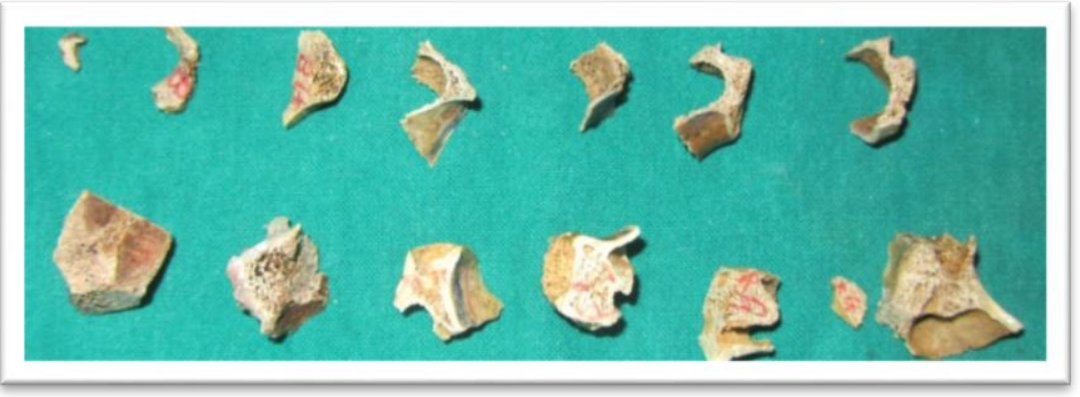

Fig. 3: Sections of the maxillary alveolar bone at the first molar region of the skulls

\section{Discussion}

The field of implant dentistry is getting its due in the last couple of decades through awareness of the patients and latest technological and surgical improvements. This is making implant dentistry as most preferred line of management for the completely edentulous and partially edentulous patients. There are so many types of implant modules which will utilize both conventional and computerized imaging technologies for the best possible placement of implants in the most suitable sites in the implant recipient areas. Modern technology has given the chance to the implantologist through advanced imaging techniques in selection of implant site and successful surgical planning with most appropriate prosthetic preferences.

As the preference of imagiologic technique differs from implantologist to implantologist an attempt is made to compare the conventional radiologic study with advanced computed tomographic techniques. This study will definitely give the clarity of various advantages between various imaging modalities so that the implantologist can choose the required imaging technique based on the requirement which is highly variable patient to patient.

The most important requisite for any saddle area in the posterior maxilla, to receive a successful implant is the height of the alveolar/crestal bone from the alveolar ridge to the floor of the maxillary sinus. ${ }^{11,12}$ The OPG is definitely helpful in getting this particular data in a simplified and economic way. Radiographic image distortion is the main disadvantage of OPG. Magnification in OPG is variable and occurs in all directions (1.1-1.7 times). ${ }^{13}$

In the present study, the magnification was considerable when the height of alveolar bone measured on OPG was compared with direct measurement by sectioning the skull. Even though, all the OPs were taken in a single OPG machine, magnification varied considerably from sample to sample. The magnification was wide ranging with average magnification of $40 \%$. There are plenty of studies in the literature regarding the magnification in the OPGs which report magnification of $20 \%$ to $40 \% .^{14,15}$ The finding in this study is on the higher side of the values reported in other studies. This may be due to the fact that the focal trough of the OPG machines is calibrated according to the anthropometric studies in the western societies where these machines are manufactured. Also, these machines are calibrated for subjects with hard and soft tissues unlike in the present study where only skulls were chosen.

Assessment of alveolar bone morphology, shape, inclination and quality is also not possible in OPG. Some 
OPG machines have tomographic functions which can give bucco-lingual width but they require multiple exposures for different areas which can make the cumulative radiation exposure much higher in case of multiple implant planning. More so ever, the bucco lingual width and mesio-distal length assessment is not accurate due to unpredictable, unequal image distortion. ${ }^{16}$

In the present study, only $15 \%$ ( $3 / 20$ measurements) of the measurements made from crest of the alveolar ridge to the floor of the maxillary sinus in the molar region were accurate with the difference under $1 \mathrm{~mm}$. This is similar to a study by Klinge et al, ${ }^{17}$ who found that only $17 \%$ of measurements made from the crest of the alveolar ridge to the most superior border of the mandibular canal were accurate within $1 \mathrm{~mm}$.

The degree of magnification varies from panoramic machine to machine. Even though every manufacturer gives the percentage of magnification for managing the percentage of error in the respective machines, the lack of feasibility in controlling the angulations between the $\mathrm{x}$-ray beams, vertical and horizontal planes of jaw bone, may contribute to distortions and deviated measurements of the various structural sites of jaw bones. The placement of metal ball stent during conventional imageology helps in overcoming the distortions and magnifications to some extent. ${ }^{18}$

In the present study, the values obtained after placement of metal ball stent were used to compare with those values obtained on computed tomograms and skull sectioning. In spite of the compensation, the values obtained on OPGs differed substantially with those obtained on computed tomograms and sectioning.

In the present study, the mean value of the height of the alveolar bone as measured in panoramic radiography was found to be $12 \mathrm{~mm}$ while, the mean value of the height of the alveolar bone as measured by sectioning the maxilla was found to be $9.3 \mathrm{~mm}$. There was statistically significant difference in the mean alveolar height between the two study groups. $(\mathrm{P}<0.001)$ Post hoc analysis showed significant higher mean alveolar height measured with OPG than that of the mean alveolar height measured by sectioning the maxilla.

While there are very few studies regarding the accuracy of various radiographic modalities compared with skull sectioning in the posterior region of the maxilla, there are plethora of such studies on mandibular posterior region stressing on position and localization of mandibular canal. ${ }^{19-21}$

Nowadays CT scans are recommended and are being routinely used for preoperative analysis of the alveolar bone due to its obvious advantages. ${ }^{22}$ It provides axial, panoramic, and cross-sectional views and additionally provides 3- dimensional images which make it easy to visualize shape, angulation and volume of the bone. ${ }^{23}$ Modern cone beam CT scanners are much more compact, economical, and user-friendly. Their image quality is much better and the scanning time and radiation exposure is much reduced. ${ }^{24}$ Further, the density and quality of bone can easily be estimated by the display of Hounsfield units over the selected region.

The high cost factor is a major deterrent for this modality. The high capital cost of the machine and availability of specially trained radiologists and technicians, tend to aggregate these facilities in urban areas only. Economic considerations and lack of availability of advanced CT equipment with desirable software applications in all dental centers is another disadvantage. The higher radiation exposure during CT procedure as compared to OPG is another disadvantage, but the additional information that can be obtained by a CT scan negates all these disadvantages and can be extremely useful to implantologist as well as the patient.

Thus in the present study, height of the alveolar bone was also measured using CT and compared with direct measurements by sectioning maxilla. In this study, the mean value of the height of the alveolar bone as measured in CT was found to be $9.4 \mathrm{~mm}$ and the mean value of the height of the alveolar bone as measured by sectioning the maxilla was found to be $9.3 \mathrm{~mm}$. Post hoc analysis showed no significant difference between the mean alveolar height measured with $\mathrm{CT}$ and that of by sectioning the maxilla.

A study reported the rates of magnifications for CT to be about $3.73 \%$ to $9.52 \%$ and $0 \%$ to $4 \% .{ }^{25}$ The mean rate of magnification for computerized tomographic images was found to be $2.04 \%$ in the present study which is comparable to previously reported results. The rate of magnification in the present study ranged from $0 \%$ to $9.3 \%$.

A study in 2010 concluded that the CT examinations with reformatted images are the only effective means of evaluating the bone volume present below the maxillary sinuses. ${ }^{13}$

In light of the above, in the present study, the values obtained by measuring the height of alveolar bone in OPG was compared to the values obtained on CT. In this study, the mean value of the height of the alveolar bone as measured in panoramic radiography was found to be $12 \mathrm{~mm}$ while, the mean value of the height of the alveolar bone as measured on computed tomographic scans was found to be $9.4 \mathrm{~mm}$. There was statistically significant difference in the mean alveolar height between the two study groups. $(p<0.001)$. Post hoc analysis showed significant higher mean alveolar height measured with OPG than that of the mean alveolar height measured on computed tomographic scans.

Another parameter which is equally important for placement of implants and its long term survival is the adequate width of the alveolar bone. Inadequate width of alveolar bone may result in dehiscence of the cortical bone and exposure of implant into the oral cavity, which could be detrimental to the survival of implant and lead to various infections as well. ${ }^{26}$ The availability of procedures like ridge augmentation and ridge split osteotomy placed even more stress on pre-surgical 
assessment of width of the alveolar bone. Two-dimensional radiographs do not provide much information on bucco-lingual position of the mandibular canal and the maxillary sinus and the shape and density of the alveolar ridges and cortical plates. ${ }^{27,28}$

In the present study, the width of the alveolar bone was assessed using computed tomographic scans and direct measurement by skull sectioning. The average width of the available crestal bone as measured in CT was found to be $10.3 \mathrm{~mm}$ while the mean value of the available crestal bone as measured directly on skull was also found to be $10.3 \mathrm{~mm}$. The details are given in the Table 5, Table 6 and Graph 5.

Paired t test was used to test the group difference and also pair wise variable differences, if any. The $p$ value for the Paired t test was 0.87 indicating the statistically insignificant difference in the mean crestal width within the two study groups.

\section{Conclusion}

With a practical point of view based on present socio-economic scenario of India, though the panoramic radiography is the preferred choice, CT is rapidly emerging as a choice of imagiological technique for implantology in most of the dental centers due to many anatomical challenges. In this study, OPG and CT were compared with the skull sectioning as gold standard, to measure the height and width of the alveolar bone from crest of the ridge to the floor of the maxillary sinus. After analyzing the results, it was concluded that:

1. Comparison of measurements in vertical bone height from crest of the alveolar ridge to the floor of the maxillary sinus was done with the OPG, CT and skull sectioning. There was significant higher mean alveolar height measured with OPG than that of the mean alveolar height measured with CT and sectioning the maxilla. No significant difference was seen between the mean alveolar height measured with CT and that of the direct measurement by sectioning the maxilla.

2. There was no significant difference in the mean crestal width as measured in $\mathrm{CT}$ and that measured directly on the skull using sectioning $(\mathrm{p}=0.87)$.

It is most clear that CT stands as the most accurate imaging modality among the common imaging modalities. Though OPG can be an excellent modality in diagnostic radiography, it stands next to $\mathrm{CT}$ when it comes to imaging for implant placement. This is mainly due to its unpredictable distortion of the visualized structures. It may not be advisable to solely rely on panoramic radiography for dental implant planning as the measurements taken on an imprecise diagnostic tool may jeopardize the whole exercise of implant placement and subsequent rehabilitation. OPG cannot give any information on the ridge width, jaw inclinations and angulations, undercuts or cross-sectional location of neurovascular bundles.
On the other hand, CT, though expensive, justifies the expense as it allows exact preoperative analysis of the available bone volume and helps to determine the appropriate position, angulation, number, and length of the planned implants. This study found the magnification of CT as compared to direct measurement by skull sectioning to be about $2 \%$, which is acceptable and statistically insignificant, whereas the panoramic radiography showed wide ranging rates of magnifications with a mean of $40 \%$.

Higher image quality with high-density resolution coupled with reduced radiation exposure and possibility to estimate the tissue density at specific sites made CT an advantageous modality in the implant planning. The accuracy and additional information elicited in CT, more than compensates for the higher economic cost and additional radiation exposure. Accurate preoperative planning may prevent procedures such as emergency sinus lift procedures and implant failures. This may prove to be beneficial to the patient in terms of reducing the cost of procedure and trauma as well.

By considering all the above factors, it may be concluded that the CT is recommended for multiple implant placement procedures and in cases of implant placement in posterior region of the maxilla especially in resorbed ridges where the higher cost and higher radiation exposure of CT scan is justified. However, OPG can still be used as a choice of implant imaging technique in short span tooth replacements and in the anterior regions of jaws with adequate quantity and quality of bone. This conforms to the principles of ALARA (as Low as Reasonably Achievable) assumption, which implies "exposure to the lowest radiation dose that allows for the necessary information".

Further studies with a larger sample size and more advanced imaging techniques like cone beam $\mathrm{CT}$ and $3 \mathrm{D}$ reconstruction for pre-operative implant imaging are required to establish available oral and maxillofacial imagiological guidelines.

\section{References}

1. Misch C: Contemporary Implant Dentistry Mosby 2008,:389-400.

2. Arun K. Garg; Dental Implant imaging: TeraRecon's Dental 3D Cone Beam Computed Tomography; System; June 2007, Volume 18, Number 6.

3. Goodacare JC et al: Clinical complications with implants and implant prostheses, J Prosth Dent 2003:89:121-32.

4. Maryam Shahbazian1, Dong Xue2, Yuqian Hu, Johan van Cleynenbreugel, ReinhildeJacobs, Spiral Computed Tomography Based Maxillary Sinus Imaging in Relation to Tooth Loss, Implant Placement and Potential Grafting Procedure, J Oral Maxillofacial Res 2010 (Jan-Mar), vol. 1, No 1, e7, p.1

5. Palmer RM, Smith BJ, Howe LC, Palmer PJ. Treatment planning: general considerations. In: Palmer RM, Smith BJ, Howe LC, Palmer PJ, editors. Implants in clinical dentistry. London: Martin Dunitz Ltd, 2002:27-34.

6. Ylikontiola L, Moberg K, Huumonen S, Soikkonen K, Oikarinen K. Comparison of three radiographic methods 
used to locate the mandibular canal in the buccolingual direction before bilateral sagittal split osteotomy. Oral Surg Oral Med Oral Pathol Oral RadiolEndod. 2002 Jun;93(6):736-42.

7. Andréia Perrellaetal, quantitative analyses of maxillaryJ Appl Oral Sci 2003; 11(3): 229-33). sinus using computed tomography;

8. Compagnone G, M CasadioBaleni, L Pagan. Comparison of radiation doses to patients undergoing standard radiographic examinations with conventional screen-film radiography, computed radiography and direct digital radiography. British Journal of Radiology (2006) 79,899-904.

9. Angelopoulos C, Thomas SL, Hechler S, Parissis N, Hlavacek M. Comparison between digital panoramic radiography and cone-beam computed tomography for the identification of the mandibular canal as part of presurgical dental implant assessment. J Oral Maxillofac Surg. 2008 Oct; 66(10):2130-5.

10. Alexandre Bahlis Luis André Mezzomo ,Daniel Boeckel ,Nilza Pereira da Costa ,Eduardo Rolim Teixeira, Accuracy of periapical radiography, panoramic radiography and computed tomography for examining the mental foramen region, Rev. odontociênc. 2010;25(3):282-7.

11. Peltola JS, Mattila M. Cross-sectional tomograms obtained with four panoramic radiographic units in the assessment of implant site measurements.

DentomaxillofacRadiol. 2004 Sep; 33(5):295-300.

12. Madrigal C, Ortega R, Meniz C, López-Quiles J. Study of available bone for interforaminal implant treatment using cone-beam computed tomography. Med Oral Patol Oral Cir Bucal. 2008 May 1; 13(5):E307-12.

13. Adam Shui-Cheong Siu et al, Imaging modalities for preoperative assessment in dental implant therapy: an overview, Hong Kong Dent J 2010;7:23-30.

14. Tronje G, Welander U, McDavid WD, Morris CR. Image distortion in rotational panoramic radiography. VI. Distortion effects in sliding systems. Acta Radiol Diagn

15. (Stockh) 1982;23:153-60.

16. Lindh C, Petersson A, KlingeB.Visualization of the mandibular canal by different radiographic techniques. Clin Oral Implants Res 1992;3:90-7.

17. Mehra A, Pai KM. Evaluation of Dimensional Accuracy of Panoramic Cross-Sectional Tomography, Its Ability to Identify the Inferior Alveolar Canal, and Its Impact on Estimation of Appropriate Implant Dimensions in the Mandibular Posterior Region. Clin Implant Dent Relat Res. 2009 Aug 6. [Epub ahead of print]

18. Klinge B, Petersson A, Maly P. Location of the mandibular canal: comparison of macroscopic findings, conventional radiography, and computed tomography. Int J Oral Maxillofacial Implants 1989; 4:327-32.

19. Babbush CA. Evaluation and selection of the endosteal implant patient. In: McKinneyRV, editor. Endosteal dental implants. St Louis: Mosby Year Book, 1991:63-74.

20. Batenburg RH, Stellingsma K, Raghoebar GM, Vissink A. Bone height measurements on panoramic radiographs: the effect of shape and position of edentulous mandibles. Oral Surg Oral Med Oral Pathol Oral RadiolEndod. 1997 Oct; 84(4):430-5.

21. Catić A, Celebić A, Valentić-Peruzović M, Catović A, Jerolimov V, Muretić I. Evaluation of the precision of dimensional measurements of the mandible on panoramic radiographs. Oral Surg Oral Med Oral Pathol Oral Radiol Endod.1998 Aug;86(2):242-8

22. Maria IveteBolzanRockenbach, Maria CarméliCorreia Sampaio1 LinoJoão Da Costa. Evaluation of Mandibular Implant Sites: Correlation between Panoramic and Linear Tomography. Braz Dent J (2003) 14(3):209-213.

23. Schwarz MS, Rothman SL, Chafetz N, Rhodes M. Computed tomography in dental implantation surgery. Dent Clin North Am 1989;33:555-97.

24. Tal H, Moses O. A comparison of panoramic radiography with computed tomography in the planning of implant surgery. Dento maxillofac Radiol. 1991 Feb; 20(1):40-2.

25. Angelopoulos C, Thomas SL, Hechler S, Parissis N, Hlavacek M. Comparison between digital panoramic radiography and cone-beam computed tomography for the identification of the mandibular canal as part of presurgical dental implant assessment. J Oral Maxillofac Surg. 2008 Oct; 66(10):2130-5.

26. . IIKayPeker ,MeryamToraman, the use of 3 different imaging methods for the localization of the mandibular canal in dental implant planning,Int J Oral Maxillofac Implants, 2008,23;463-70.

27. Gröndahl K, Ekestubbe A, Gröndahl HG, Johnsson T. Reliability of hypocycloidal tomography for the evaluation of the distance from the alveolar crest to the mandibular canal. DentomaxillofacRadiol 1997;20:200-4.

28. Palmer RM, Smith BJ, Howe LC, Palmer PJ. Treatment planning: general considerations. In: Palmer RM, Smith BJ, Howe LC, Palmer PJ, editors. Implants in clinical dentistry. London: Martin Dunitz Ltd, 2002:27-34.

29. Ylikontiola L, Moberg K, Huumonen S, Soikkonen K, Oikarinen K. Comparison of three radiographic methods used to locate the mandibular canal in the buccolingual direction before bilateral sagittal split osteotomy. Oral Surg Oral Med Oral Pathol Oral RadiolEndod. 2002 Jun; 93(6):736-42. 